\title{
Decreased expression of miR-490-3p in colorectal cancer predicts poor prognosis and promotes cell proliferation and invasion by targeting RAB14
}

\author{
BO WANG ${ }^{1 *}$, MUJUN YIN $^{1}{ }^{*}$, CHENG CHENG $^{2}$, HONGPENG JIANG $^{1}$, \\ KEWEI JIANG $^{1}$, ZHANLONG SHEN ${ }^{1}$, YINGJIANG YE $^{1}$ and SHAN WANG ${ }^{1}$ \\ ${ }^{1}$ Department of Gastroenterological Surgery, Laboratory of Surgical Oncology, \\ Beijing Key Laboratory of Colorectal Cancer Diagnosis and Treatment Research, \\ Peking University People's Hospital, Beijing 100044; ${ }^{2}$ Department of General Surgery, \\ First Hospital of Qinhuangdao, Hebei 066000, P.R. China
}

Received February 14, 2018; Accepted May 16, 2018

DOI: 10.3892/ijo.2018.4444

\begin{abstract}
Growing evidence indicates a potential role for miR-490-3p in tumorigenesis. However, its function in colorectal carcinoma (CRC) remains undefined. In this study, miR-490-3p was markedly downregulated in fifty colorectal cancer tissue samples compared with the corresponding adjacent non-cancerous specimens, by reverse transcriptionquantitative polymerase chain reaction (RT-qPCR). The expression levels of miR-490-3p were closely associated with tumor differentiation and distant metastasis. In addition, both Kaplan-Meier and multivariate analyses indicated CRC patients with elevated miR-490-3p amounts had prolonged overall survival. Overexpression of miR-490-3p markedly reduced proliferation, colony formation and invasion in CRC cells by enhancing apoptosis and promoting $\mathrm{G} 2 / \mathrm{M}$ phase arrest. Furthermore, ectopic expression of miR-490-3p resulted in decreased expression of RAB14, which was directly targeted by $\mathrm{miR}-490-3 \mathrm{p}$, as shown by the dual-luciferase reporter gene assay. Finally, in a nude mouse model, miR-490-3p overexpression significantly suppressed the growth of CRC cells. The above results indicated that miR-490-3p might constitute a prognostic indicator and a novel molecular target for miRNAbased CRC therapy.
\end{abstract}

Correspondence to: Professor Yingjiang Ye or Professor Zhanlong Shen, Department of Gastroenterological Surgery, Laboratory of Surgical Oncology, Beijing Key Laboratory of Colorectal Cancer Diagnosis and Treatment Research, Peking University People's Hospital, 11 Xizhimen South Street, Beijing 100044, P.R. China

E-mail: yeyingjiang@pkuph.edu.cn

E-mail: shenlong1977@163.com

${ }^{*}$ Contributed equally

Key words: miR-490-3p, RAB14, colorectal cancer

\section{Introduction}

Colorectal carcinoma (CRC) is among the top three malignancies with increasing rates of morbidity and mortality worldwide (1). Despite significant advances in cancer treatment, individuals with relapse or metastases still exhibit poor prognosis (2). Therefore, the mechanisms of CRC progression require further investigation.

$\mathrm{RAB}$ proteins regulate diverse pathways in intracellular membrane trafficking and dynamics (3). Distinct RAB proteins exert different effects. For example, RAB-1 and RAB-2 modulate the innate immunity, neuron maturation and vesicle trafficking (4). Meanwhile, RAB14 is overexpressed in multiple human malignancies, including ovarian (5), non-small cell lung $(6)$, breast $(7)$ and gastric $(8,9)$ cancer. RAB14 appears to act as an oncogene in human tumorigenesis and metastasis. However, how RAB14 affects CRC tumorigenesis remains unclear. Therefore, identifying and characterizing RAB14 is essential for understanding its roles in the pathogenesis of CRC.

MicroRNAs (miRNAs) constitute important mediators, which regulate target genes via binding to the 3'-untranslated region (UTR) of mRNAs (10). Multiple miRNAs are aberrantly expressed in CRC, which promotes malignant behavior $(11,12)$. Previous findings by the authors demonstrated that the overexpression of miR-194 or miR-217 significantly decreased the proliferation and invasion of CRC cells. miR-199b might target Sirtuin 1 to suppress the metastasis of CRC to the liver (13-15). Meanwhile, miR-490-3p promotes cell proliferation and metastasis in liver cancer by targeting ERGIC and Golgi 3 (16) while inhibiting other malignant tumors, including gastric cancer $(17,18)$, ovarian cancer (19), breast cancer (20) and osteosarcoma (21). Although Xu et al (22) and Zheng et al (23) reported that miR-490-3p was downregulated in colorectal carcinoma, the mechanism associated with its role is not completely elucidated. 
The present study aimed to assess whether RAB14 is directly targeted by miR-490-3p, and evaluate the role of the miR-490-3p/RAB14 pathway in the progression of CRC.

\section{Materials and methods}

Cells and human tissue specimens. Human colorectal cancer cells, SW480, SW620, RKO, HT29, HCT116 and LoVo, were purchased from the American Type Culture Collection (Manassas, VA, USA). NCM460 cells were from INCELL Corporation LLC (San Antonio, TX, USA). HT29, LoVo, HCT116 and RKO cells were cultured in RPMI-1640. SW480 and SW620 were cultured in Leibovitz's L-15 medium. All cells were cultured at $37^{\circ} \mathrm{C}$ in a humidified environment containing $5 \% \mathrm{CO}^{2}$, and the medium was supplemented with $10 \%$ fetal bovine serum (FBS, Gibco; Thermo Fisher Scientific, Inc., Waltham, MA, USA), $100 \mathrm{U} / \mathrm{ml}$ penicillin (Sigma-Aldrich; Merck KGaA), and $100 \mu \mathrm{g} / \mathrm{ml}$ streptomycin (Sigma-Aldrich; Merck KGaA).

A total of 90 patients with CRC were involved in the present study. Accordingly, 90 human colorectal cancerous specimens and the corresponding adjacent non-cancerous tissues were collected during operations at Peking University People's Hospital during January 2013 to December 2016 in subjects that underwent coloproctectomy according to the National Comprehensive Cancer Network guidelines for colon/rectal carcinoma (https://www.nccn.org/professionals/physician_gls/pdf/colon.pdf or https://www.nccn. org/professionals/physician_gls/pdf/rectal.pdf, version 1 . 2013-2016). The samples were kept at $-80^{\circ} \mathrm{C}$ until use. Written informed consent was provided by all patients prior to sample collection. The present study received approval from the Research Ethics Committee of Peking University (Beijing, China).

Reverse transcription-quantitative polymerase chain reaction ( $R T-q P C R)$. The purification of total RNA and RT-qPCR were performed with PrimeScript RT reagent kit and SYBR Green PCR Master Mix, respectively (Takara Bio, Inc., Otsu, Japan) as described by the manufacturer. The thermocycling conditions were $5 \mathrm{sec}$ at $95^{\circ} \mathrm{C}$ and $30 \mathrm{sec}$ at $60^{\circ} \mathrm{C}$. The data were analyzed using the $2^{-\Delta \Delta \mathrm{Cq}}$ method as previously described by Livak and Schmittgen (24) and Wang et al (15). GAPDH and U6 RNA were employed as references for mRNAs and miRNAs, respectively. The primers used were: miR-490-3p forward, 5'-CAACCTGGAGGACTCCATGCTG-3'; U6 forward, 5'-CTCGCTTCGGCAGCACA-3'; RAB14 forward, 5'-CGCTCGAGATGGCAACTGCACCATACAAC-3' and reverse, 5'-CGGAATTCCTAGCAGCCACAGCCTTCTC-3'; GAPDH forward, 5'-CCCCGGTTTCTATAAATTGAGC-3' and reverse, 5'-CACCTTCCCCATGGTGTCT-3'. The universal primer from Takara Bio, Inc. was used as the reverse primer for miR-490-3p and U6.

Cell transfection or infection. The miRNAs and siRNAs were synthesized by Guangzhou RiboBio Co., Ltd., (Guangzhou, China). RAB14 plasmids were manufactured by Invitrogen (Thermo Fisher Scientific, Inc.). Transfection was carried out with Lipofectamine ${ }^{\circledR} 2000$ (Invitrogen; Thermo Fisher Scientific, Inc.). RAB14 siRNA, miR-490-3p mimics and respective negative controls were used at $50 \mathrm{nM}$ for transient transfection. Lentiviral vectors $(3 \mathrm{E}+8 \mathrm{TU} / \mathrm{ml})$ (Shanghai GeneChem Co., Ltd., Shanghai, China) expressing miR-490-3p (LV-miR-490-3p) or the negative control sequence were employed to infect CRC cells for in vivo experiments. The oligonucleotide sequences for transfection were: miR-490-3p mimics, 5'-CAACCUGGAGGACUCCAUGCUG-3'; miR-NC, 5'-UUUGUACUACACAAAAGUACUG-3' and RAB14 siRNA, 5'-TGCAAGGAATCTCACCAAT-3'.

Western blot assay. Cell lysis was performed with hypotonic lysis buffer (Thermo Fisher Scientific, Inc.). Equal quantities of protein $(20 \mu \mathrm{g} /$ lane $)$ were resolved by $10 \%$ or $15 \%$ SDS-PAGE and electro-transferred onto nitrocellulose membranes (Pall Corporation, Pensacola, FL, USA). The detailed description of the assay was recently reported (14). Briefly, the membranes were blocked with 5\% non-fat milk powder in Tris-buffered saline containing $0.1 \%$ Tween-20 and probed with primary antibodies overnight at $4^{\circ} \mathrm{C}$. Following incubation with secondary antibodies in room temperature, the bands were visualized using enhanced chemiluminescence (Pierce; Thermo Fisher Scientific Inc.) and imaged using a ChemiDoc ${ }^{\mathrm{TM}}$ XRSC system (Bio-Rad Laboratories, Inc., Hercules, CA, USA). The primary antibodies used were: anti-GAPDH (1:1,000; cat. no. 2118; Cell Signaling Technology, Inc., Danvers, MA, USA) and anti-RAB14 (1:500; cat. no. ab28639; Abcam, Cambridge, UK). The secondary antibody used was anti-rabbit $\operatorname{IgG}(1: 5,000$; cat. no. ab286397074; Cell Signaling Technology, Inc.).

Cell proliferation and colony formation assay. Cell proliferation was assessed using the Cell Counting Kit-8 assay. After the cells (SW480 or SW620) were seeded into 96-well plates at 2,000 cells/well, incubation was carried out at $37^{\circ} \mathrm{C}$ for $48 \mathrm{~h}$. Viable cells were quantified at various times at $450 \mathrm{~nm}$ on a microplate reader (Bio-Rad Laboratories, Inc.). Sextuplicate assays were performed for three times.

To assess the ability of the cells to form colonies, the cells were seeded into 6-well plates and cultured for two weeks at $37^{\circ} \mathrm{C}$ following transfection. Next, the cells were washed with PBS and fixed with $4 \%$ paraformaldehyde then subjected to staining with $0.1 \%$ crystal violet for $20 \mathrm{~min}$ at room temperature. Duplicate assays were carried out three times.

Cell cycle and apoptosis analysis. To assess cell cycle distribution, the cells (SW480 or SW620) were stained with the BD Cycletest ${ }^{\mathrm{TM}}$ plus DNA kit (BD Biosciences, Franklin Lakes, NJ, USA) as suggested by the manufacturer. To detect apoptosis, the transfected cells $(72 \mathrm{~h})$ were incubated with the Alexa Fluor ${ }^{\circledR} 488$ Annexin V/Dead cell apoptosis kit (Invitrogen; Thermo Fisher Scientific, Inc.). Apoptosis analysis was performed according to the manufacturer's instructions. The temperature of incubation was different depending on the steps undertaken. Data analysis was carried out with FlowJo (version 7.0; Tree Star, Inc., Ashland, OR, USA) on a flow cytometer (BD Biosciences).

Cell invasion assay. Cell invasion assay was performed using the Transwell method. Cancer cells (SW480 or 
A

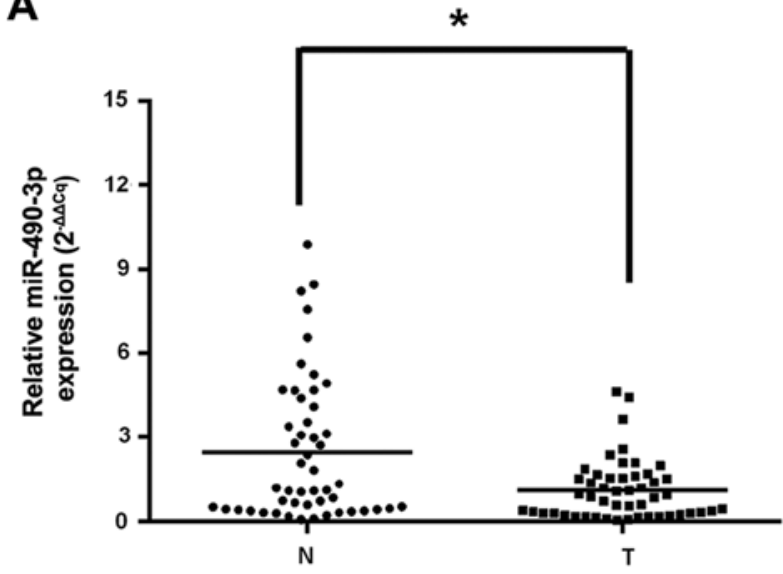

B

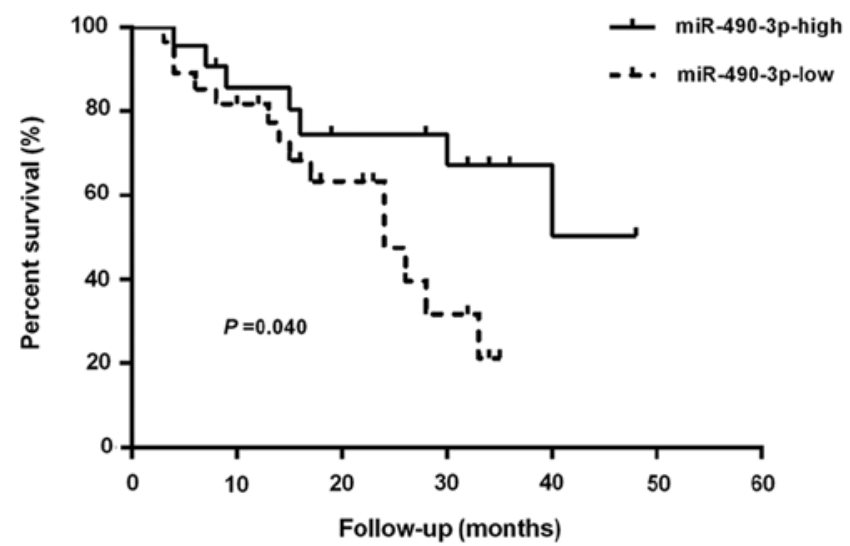

Figure 1. miR-490-3p expression in CRC tissue specimens and clinical significance. (A) Relative expression levels of miR-490-3p in human CRC (n=50) and CRN ( $n=50)$ tissue specimens, assessed by reverse transcription-quantitative polymerase chain reaction. CRC, colorectal carcinoma samples; CRN, matched adjacent non-cancerous colorectal tissue specimens. (B) Kaplan-Meier survival curves of CRC cases with elevated ( $\mathrm{n}=22$ ) and reduced ( $\mathrm{n}=28) \mathrm{miR}-490-3 \mathrm{p}$. ${ }^{*} \mathrm{P}<0.05$ assessed using log-rank test. CRC, colorectal carcinoma; miRNA, microRNA; N, adjacent normal colorectal samples; T, CRC tissue specimens.

SW620) were added to the upper chambers (24-well plate, pore size, $8 \mu \mathrm{m}$; Corning Incorporated, Corning, NY, USA) with Matrigel pre-coated membranes. Leibovitz's L-15 medium with $30 \%$ FBS was employed as a chemoattractant in the lower chambers. The Transwell plate was incubated for 2 days. The cells that passed through the membrane were washed with PBS. The cells were fixed with $4 \%$ paraformaldehyde for $20 \mathrm{~min}$ and stained with $0.1 \%$ crystal violet for $20 \mathrm{~min}$ at room temperature. Subsequently, the cells were counted and imaged using an inverted microscope (magnification, x200; Leica DM IL LED; Leica Microsystems $\mathrm{GmbH}$, Wetzlar, Germany). Duplicate assays were carried out three times.

Luciferase reporter assay. The miR-490-3p-binding site in RAB14 was predicted using TargetScan 7.1 (http://www. targetscan.org/), microRNA (http://www.microrna.org/) and miRDB (http://www.mirdb.org/). SW480 cells in 24-well plates were co-transfected with luciferase plasmids (wild-type or mutant, $500 \mathrm{ng} /$ well) for RAB14 and miR-490-3p or NC mimics as directed by the manufacturer (RiboBio Co., Ltd.). The samples were incubated for $48 \mathrm{~h}$ at $37^{\circ} \mathrm{C}$, and luciferase activities were assessed using the Dual-Luciferase reporter assay system (Promega Corporation, Madison, WI, USA). The data were reported as a ratio of Firefly to Renilla luciferase activity.

Xenograft mice model. A total of 8 BALB/c-nude mice (female, 6-weeks-old; Vital River Laboratories, Beijing, China) were randomly allocated to two groups with 4 mice in each group to examine tumorigenicity. In this assay, $200 \mu \mathrm{l}$ cell suspensions $\left(1 \times 10^{7} \mathrm{SW} 480\right.$ cells) were subcutaneously administered to the right flank of each mouse. The volume of the tumors was assessed at 4-day intervals using the formula: $\mathrm{V}=0.5 \mathrm{x} \mathrm{L}$. The animals were scarified 40 days after cell inoculation and the tumors were extracted for volume and weight measurements. All animal experiments received approval from the Animal Research Committee of the Peking University People's Hospital (Beijing, China). The mice were handled following guidelines provided by the Institutional and Animal Care and Use Committee.

Statistical analysis. The data are expressed as the mean \pm standard deviation and were assessed with SPSS (version 20.0; SPSS, Inc., Chicago, IL, USA). Student's t-test was employed for group comparisons while LSD t-test was performed for multiple comparisons. The associations of miR-490-3p levels with clinicopathologic parameters in CRC were evaluated using the Pearson $\chi^{2}$ test. The Kaplan-Meier method was employed to assess overall survival with group differences determined by the log-rank test. Cox regression analysis was used for multivariate analysis. The association between the expression of miR-490-3p and RAB14 was evaluated using the Spearman's correlation. $\mathrm{P}<0.05$ indicated statistical significance.

\section{Results}

Low miR-490-3p levels indicate poor prognosis in CRC. RT-qPCR was employed to assess the levels of miR-490-3p, which were markedly reduced in CRC tissue specimens compared with adjacent normal colorectal samples (Fig. 1A). In addition, decreased miR-490-3p levels were highly correlated with late tumor-node metastasis (TNM) stage and lymph node metastasis, but not age, sex, tumor size, tumor differentiation and distant metastasis (Table I). Using the Kaplan-Meier method, it was identified that CRC cases expressing low levels of miR-490-3p had a reduced median survival compared with those exhibiting elevated miR-490-3p expression (Fig. 1B). Cox's multivariate analysis demonstrated that distant metastasis was closely associated with overall survival in CRC, and it was an independent predictor of survival (Table II).

miRNA-490-3p suppresses the proliferation of CRC cells in vitro and in vivo. As CRC cells express low levels of miR-490-3p (Fig. 2A), gain-of-function studies of miR-490-3p were performed by transfecting miR-490-3p mimics into 
Table I. Association between miR-490-3p expression and clinicopathologic characteristics in patients with colorectal cancer.

\begin{tabular}{|c|c|c|c|c|}
\hline \multirow[b]{2}{*}{ Parameters } & \multicolumn{4}{|c|}{ miR-490-3p expression } \\
\hline & $\operatorname{High}(\mathrm{n}=22)$ & Low $(n=28)$ & Total $(\mathrm{n}=50)$ & P-value \\
\hline \multicolumn{5}{|l|}{ Age, years } \\
\hline$\leq 60$ & 7 & 11 & 18 & \multirow[t]{2}{*}{0.585} \\
\hline$>60$ & 15 & 17 & 32 & \\
\hline \multicolumn{5}{|l|}{ Sex } \\
\hline Female & 11 & 14 & 25 & \multirow[t]{2}{*}{1.000} \\
\hline Male & 11 & 14 & 25 & \\
\hline \multicolumn{5}{|l|}{ Tumor size, $\mathrm{cm}$} \\
\hline$\leq 2$ & 9 & 11 & 20 & \multirow[t]{2}{*}{0.907} \\
\hline$>2$ & 13 & 17 & 30 & \\
\hline \multicolumn{5}{|c|}{ Tumor differentiation } \\
\hline Well/moderate & 12 & 14 & 26 & \multirow[t]{2}{*}{0.750} \\
\hline Poor & 10 & 14 & 24 & \\
\hline \multicolumn{5}{|l|}{ TNM stage } \\
\hline $\mathrm{I}+\mathrm{II}$ & 14 & 9 & 23 & \multirow[t]{2}{*}{$0.027^{\mathrm{a}}$} \\
\hline $\mathrm{III}+\mathrm{IV}$ & 8 & 19 & 27 & \\
\hline \multicolumn{5}{|c|}{ Lymph node metastasis } \\
\hline Positive & 7 & 18 & 25 & \multirow[t]{2}{*}{$0.023^{\mathrm{a}}$} \\
\hline Negative & 15 & 10 & 25 & \\
\hline \multicolumn{5}{|l|}{ Distant metastasis } \\
\hline Positive & 5 & 12 & 17 & \multirow[t]{2}{*}{0.136} \\
\hline Negative & 17 & 16 & 33 & \\
\hline \multicolumn{5}{|c|}{ Vascular infiltration } \\
\hline Positive & 10 & 14 & 24 & \multirow[t]{2}{*}{0.750} \\
\hline Negative & 12 & 14 & 26 & \\
\hline
\end{tabular}

${ }^{\text {aP }}<0.05$. miR, miRNA; TNM, tumor-node metastasis.

Table II. Multivariate analysis of factors that are associated with overall survival in patients with colorectal cancer.

\begin{tabular}{llc}
\hline \multirow{2}{*}{ Variables } & \multicolumn{2}{c}{ Multivariate analysis } \\
\cline { 2 - 3 } & \multicolumn{1}{c}{ HR $(95 \% \mathrm{CI})$} & P-value \\
\hline Age & $1.021(0.989-1.054)$ & 0.197 \\
Sex & $0.560(0.165-1.900)$ & 0.352 \\
Tumor size & $0.956(0.321-2.847)$ & 0.936 \\
Tumor differentiation & $1.292(0.467-3.572)$ & 0.622 \\
TNM stage & $9.829(0.724-133.442)$ & 0.086 \\
Lymph node metastasis & $0.257(0.036-1.838)$ & 0.274 \\
Distant metastasis & $6.538(1.615-26.473)$ & $0.009^{\mathrm{a}}$ \\
miR-490-3p & $0.707(0.205-2.439)$ & 0.583 \\
RAB14 & $2.019(0.658-6.202)$ & 0.220 \\
\hline
\end{tabular}

${ }^{\mathrm{a}} \mathrm{P}<0.05$. CI, confidence interval; HR, hazard ratio;miR, miRNA; TNM, tumor-node metastasis.

cells (Fig. 2B). After upregulating miR-490-3p, proliferative ability was markedly repressed in SW480 and SW620 cells (Fig. 2C). Additionally, SW480 and SW620 cells transfected with miR-490-3p mimics formed markedly fewer colonies compared with the NC controls (Fig. 2D). To further confirm these findings, an in vivo study was carried out where SW480 cells were infected with LV-miR-490-3p and LV-miR-NC. Tumors from the LV-miR-490-3p-infected cells grew much more slowly compared with the LV-miR-NC group (Fig. 2E and F). In addition, miR-490-3p levels in the LV-miR-490-3pinfected tumors were higher compared with the control values (Fig. 2G).

miR-490-3p induces cancer cell cycle arrest. miR-490-3p overexpression resulted in the accumulation of SW480 $(1.235 \pm 0.078$ vs. $5.175 \pm 0.106 \%, \mathrm{P}<0.01)$ and $\mathrm{SW620}$ $(2.655 \pm 0.332$ vs. $8.110 \pm 0.665 \%, \mathrm{P}<0.01)$ cells in the $\mathrm{G} 2-\mathrm{M}$ phase (Fig. 3A). However, miR-490-3p overexpression seemed to have no significant effects on apoptosis in CRC cells (Fig. 3B).

miR-490-3p suppresses the invasive ability of CRC cells. To assess the function of miR-490-3p in CRC metastasis, miR-490-3p mimics were transfected into SW480 and SW620 cells. Transwell assays indicated that miR-490-3p overexpression decreased the invasive abilities of SW480 and SW620 cells (Fig. 3C). 
A

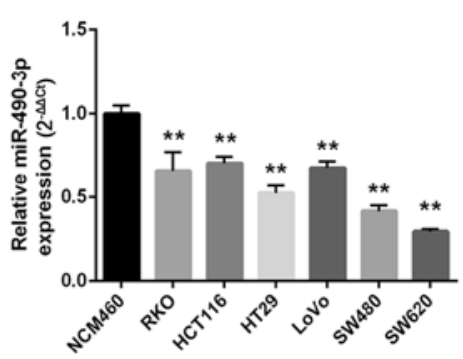

B

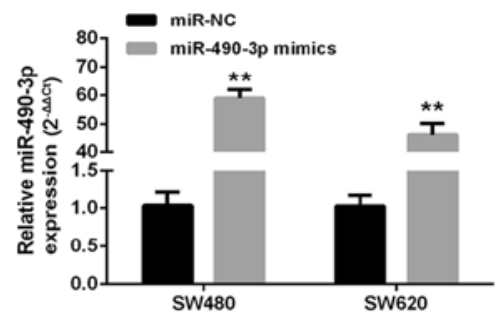

C
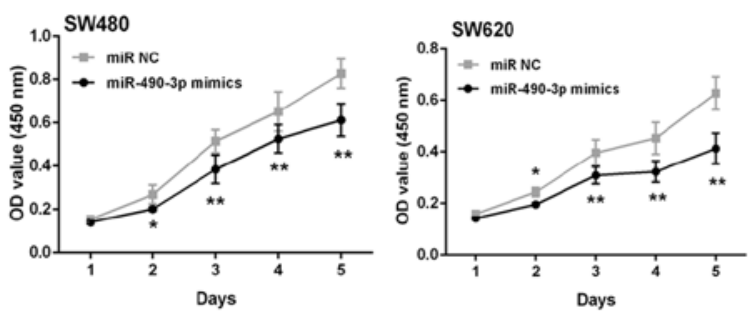

D

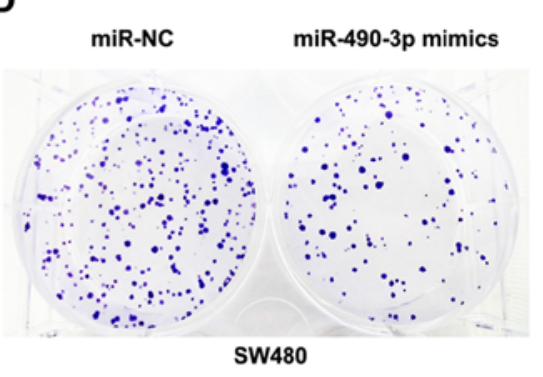

SW480

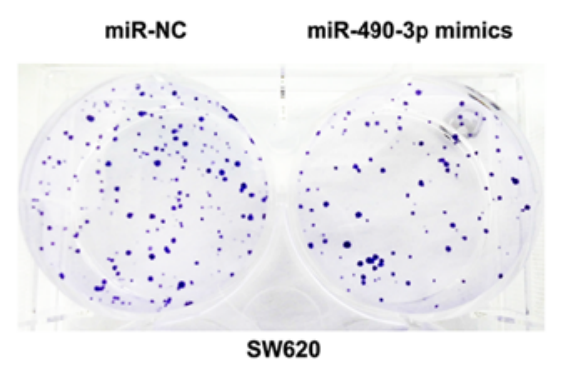

F

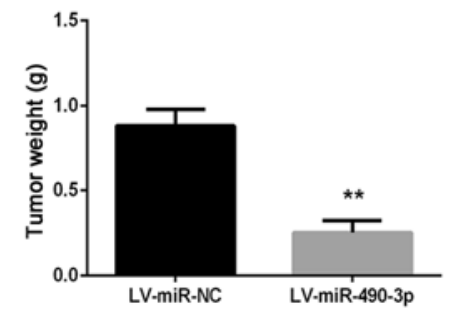

G

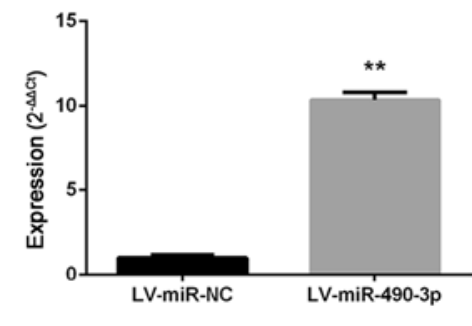

Figure 2. miR-490-3p represses the growth of CRC cells in vitro and in mice. (A) Relative miR-490-3p expression levels in 6 CRC cell lines and the normal colorectal cell line, NCM460. Mean gene expression in NCM460 was considered to be 1. (B) Relative expression levels of miR-490-3p upon transfection with miR-490-3p mimics or miR-NC as evaluated by reverse transcription-quantitative polymerase chain reaction. Mean miRNA levels in miR-NC were considered to be 1. (C) Growth curves of SW480 and SW620 cells following the overexpression of miR-490-3p as assessed by Cell Counting Kit- 8 assay. (D) Colony formation ability following the overexpression of miR-490-3p. (E) Effects of miR-490-3p on the growth of xenograft CRC tumor in mouse models. Infected SW480 cells were injected into nude mouse models. Tumor growth curves were generated by assessing tumor volumes at 4-day intervals. (F) Following the sacrifice of the mice, the weight of the tumors was quantified. Data are expressed as the mean \pm standard deviation. (G) Relative miR-490-3p expression levels in extracted tumors, normalized to the reference control U6 RNA. ${ }^{*} \mathrm{P}<0.05$, ${ }^{* *} \mathrm{P}<0.01$. CRC, colorectal carcinoma; miRNA, microRNA; NC, negative control.

miR-490-3p directly targets RAB14 by interacting with its 3'UTR. To explore the mechanisms by which miR-490-3p suppresses the malignant behavior of CRC cells, TargetScan 7.1, microRNA and miRDB were employed to identify potential miR-490-3p targets. The databases revealed that the miRNA responsive element within the 3'UTR of RAB14 is a putative miR-490-3p target (Fig. 4A). Therefore, dual-luciferase reporters comprising the 3'-UTR of RAB14 with wild-type and mutated seed sequence were cloned (Fig. 4A). Following the co-transfection of miR-490-3p or NC mimics with RAB14-UTR-WT or RAB14-UTR-MUT plasmid into SW480 cells, the relative luciferase activity in the wild-type group was markedly reduced, while the mutant group exhibited no significant changes (Fig. 4B). In addition, the effects of miR-490-3p on endogenous RAB14 expression were assessed by RT-qPCR and immunoblotting. Interestingly, the overexpression of miR-490-3p markedly reduced the levels of RAB14 mRNA (Fig. 4C) and protein (Fig. 4D).
Association of miR-490-3p with RAB14 expression in colorectal tissue specimens. Next, the association of miR-490-3p with RAB14 expression was examined in CRC and normal colorectal tissue specimens. RAB14 levels were markedly elevated in CRC tissues compared with noncancerous specimens $(\mathrm{P}<0.01$; Fig. 5A). Notably, an inverse correlation was obtained between miR-490-3p and RAB14 levels in CRC $(r=-0.3700, P<0.05)$ and normal $(r=-0.3202$, $\mathrm{P}<0.01$ ) tissues by Pearson's correlation analysis (Fig. 5B and C).

Upregulation of RAB14 counteracts the inhibitory effects of miR-490-3p on malignant behavior in SW480 cells. To confirm the role of RAB14 in miR-490-3p associated anticancer process, rescue experiments were carried out by upregulating RAB14 after transfecting miR-490-3p mimics. The overexpression of miR-490-3p and RAB14 had no significant effects on proliferation (Fig. 6A) and invasion (Fig. 6B) in SW480 cells. 

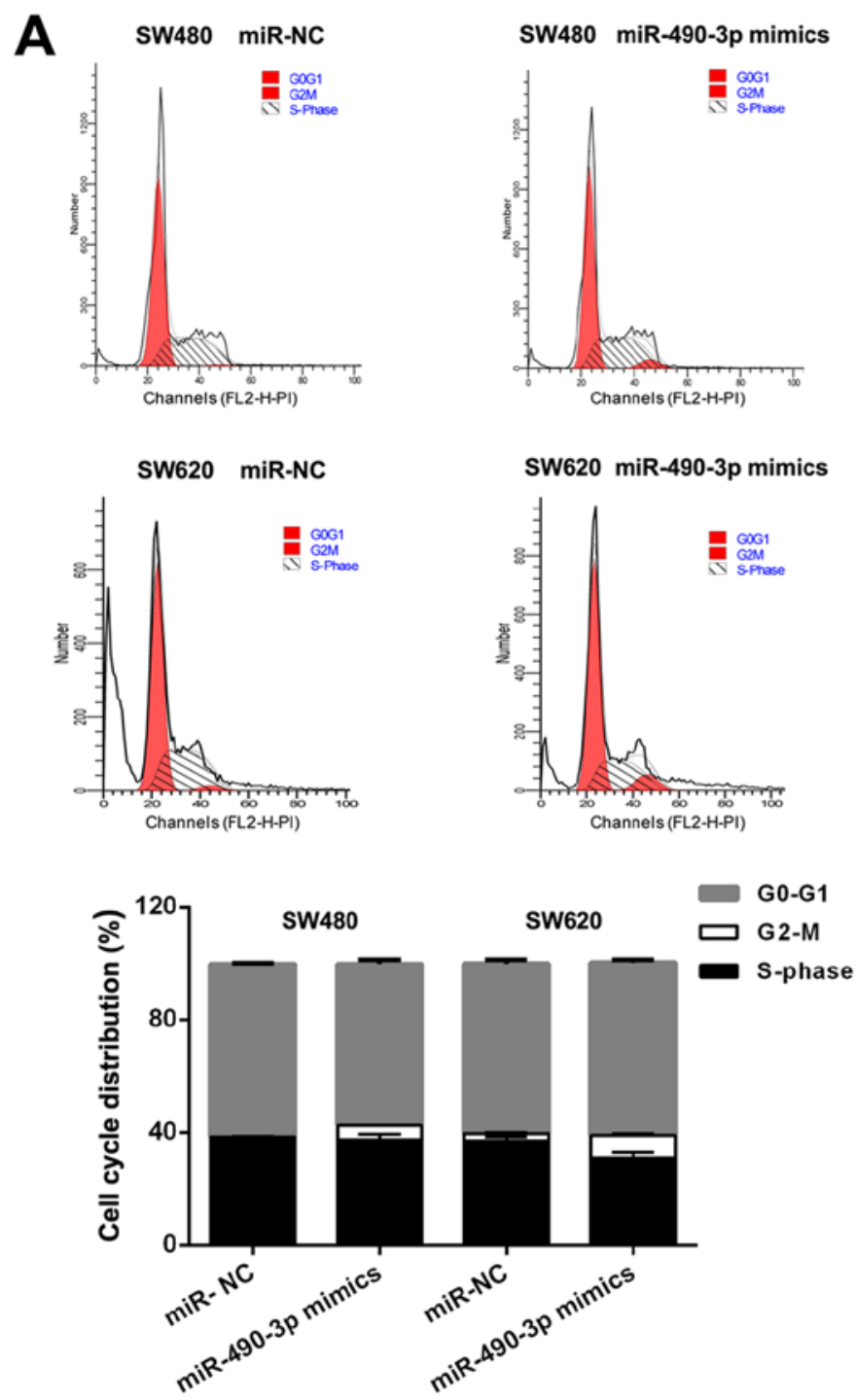

C
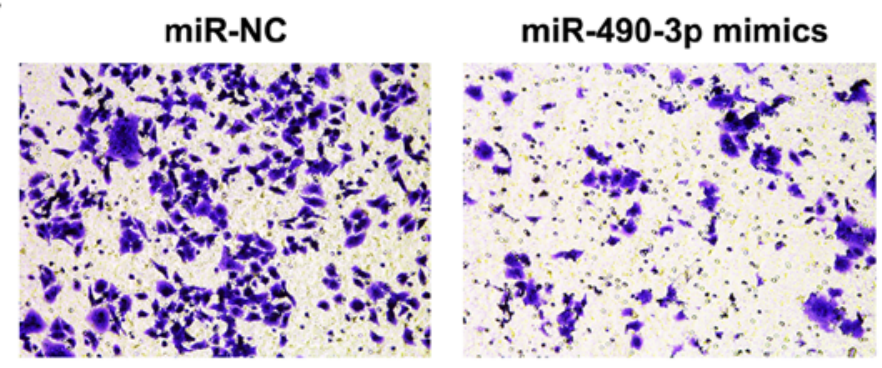

\section{SW480}

miR-NC

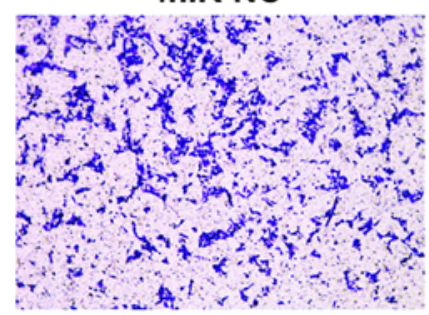

SW480 miR-490-3p mimics

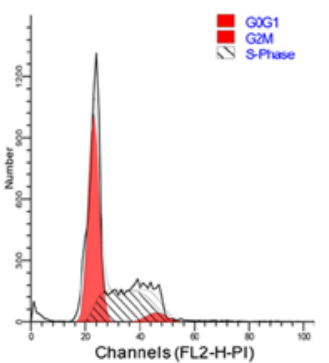

SW620 miR-490-3p mimics

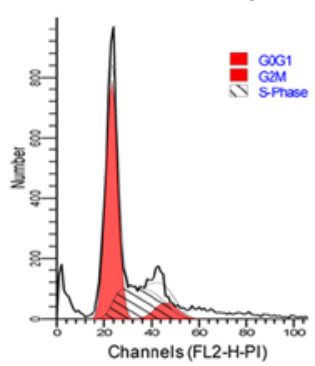

B
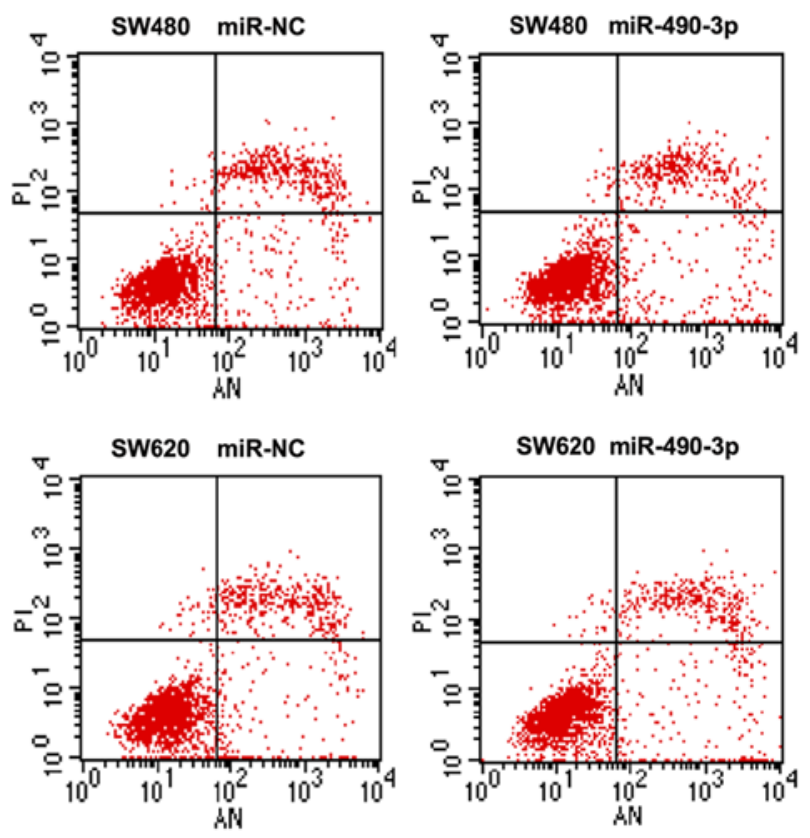
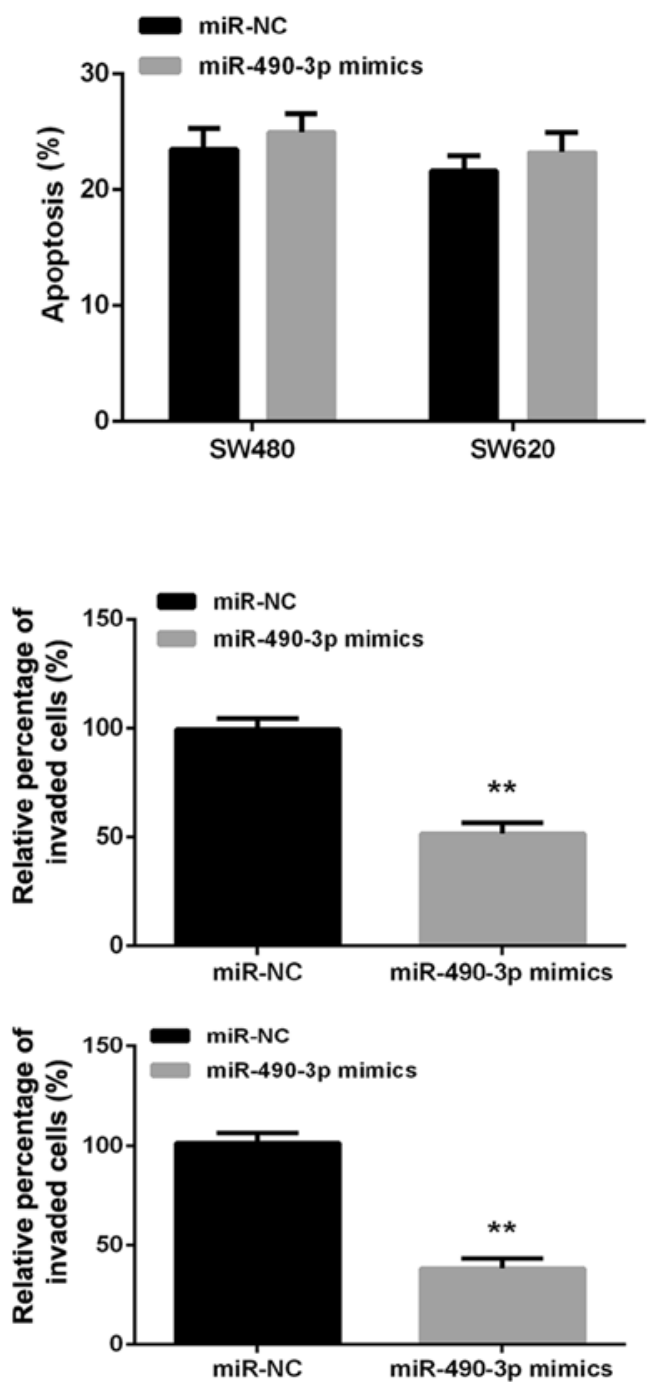

Figure 3. Ectopic miR-490-3p expression promotes G2/M phase arrest and inhibits invasion in CRC cells. (A) Cell cycle distribution was assessed by flow cytometry following treatment with mimics. miR-490-3p promoted cell cycle arrest at the G2-M phase. (B) Transfection with miR-490-3p mimics had no effects on apoptosis in CRC cells as indicated by flow cytometric analysis. (C) Transwell assay was performed to evaluate the invasive abilities of SW480 and SW620 cells following miR-490-3p upregulation. The stained cells in the lower chambers were counted under a microscope (magnification, $\mathrm{x} 200$ ). ${ }^{* *} \mathrm{P}<0.01$. CRC, colorectal carcinoma; miRNA, microRNA; NC, negative control. 
A

\begin{tabular}{|c|c|c|}
\hline & & mutant \\
\hline Position $177-183$ of & $5^{\prime}$ & ...ACAACUAGAUGUAAUCAGGUUAU... \\
\hline RAB14 3' UTR & & $\|||||$ \\
\hline hsa-miR-490-3p & $3^{\prime}$ & 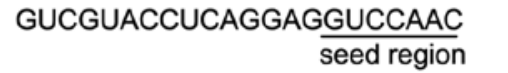 \\
\hline
\end{tabular}

B

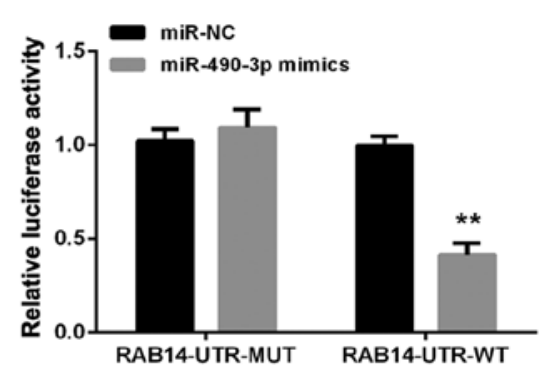

C
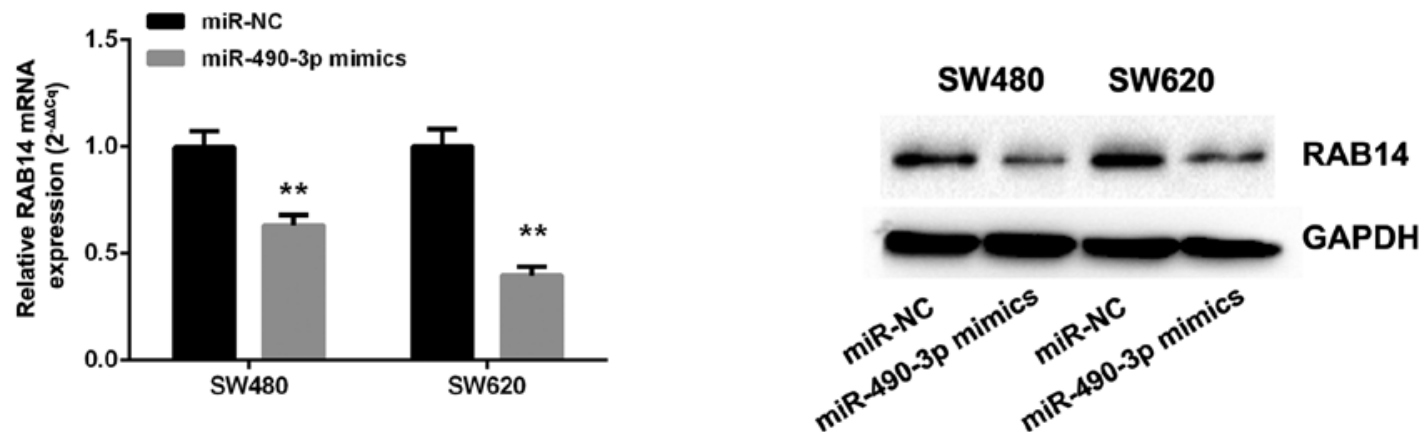

Figure 4. RAB14 is directly targeted by miR-490-3p. (A) Prediction of the binding site of human miR-490-3p to the 3'-UTR of RAB14 by TargetScan 7.1. The mutated binding site of miR-490-3p in the 3'UTR of RAB14 is illustrated. (B) A reporter plasmid comprising wild-type or mutant 3'UTR of RAB14 was co-transfected with miR-490-3p mimics or miR-NC. Relative luciferase activity was determined as firefly luciferase activity normalized to that of Renilla luciferase. Effects of miR-490-3p upregulation on RAB14 expression at (C) mRNA and (D) protein levels. Gene and protein expression levels were evaluated 48 and $72 \mathrm{~h}$ after transfection by reverse transcription-quantitative polymerase chain reaction and immunoblotting, respectively. ${ }^{* *} \mathrm{P}<0.01$. miR, miRNA; MUT, mutated; NC, negative control; UTR, untranslated region; WT, wild-type.

A

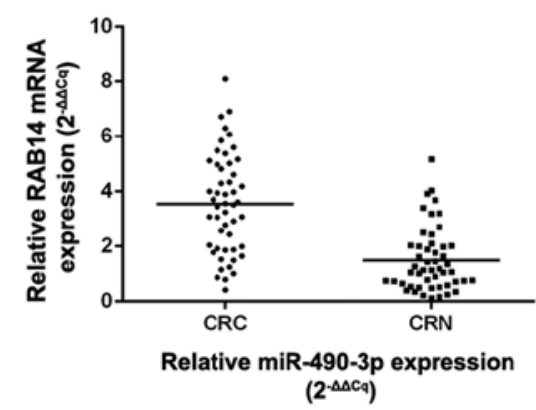

B

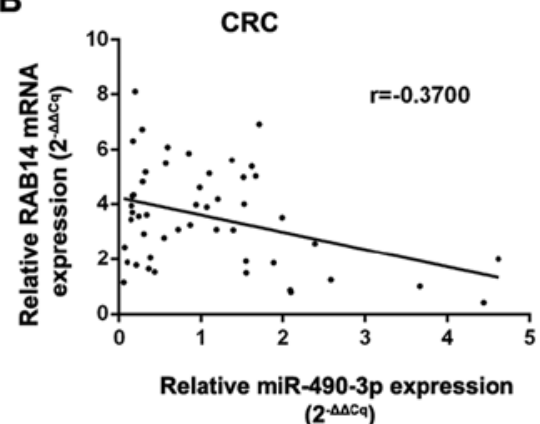

C

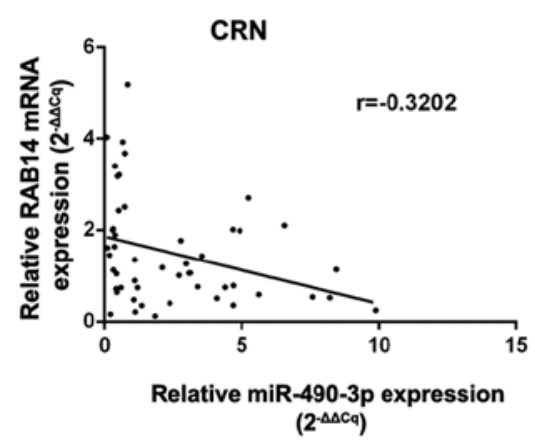

Figure 5. RAB14 is upregulated in CRC and inversely correlated with miR-490-3p expression in tissue samples. (A) Increased expression of RAB14 was detected in CRC specimens compared with adjacent normal counterparts by reverse transcription-quantitative polymerase chain reaction. Pearson's correlation analysis of the association of RAB14 expression with miR-490-3p in (B) CRC and (C) non-cancerous samples. ${ }^{* *} \mathrm{P}<0.01$. CRC, colorectal carcinoma; CRN, adjacent normal colorectal samples; miR, miRNA.

A

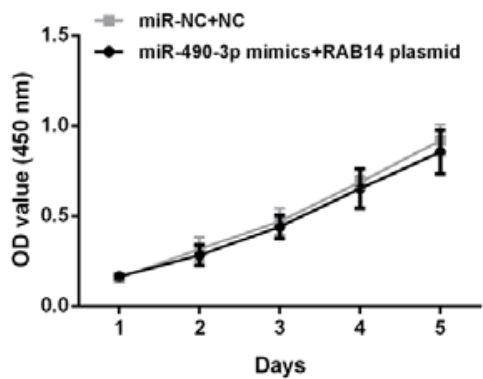

B

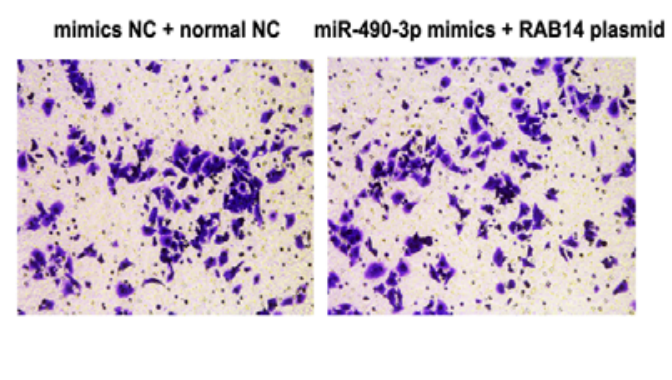

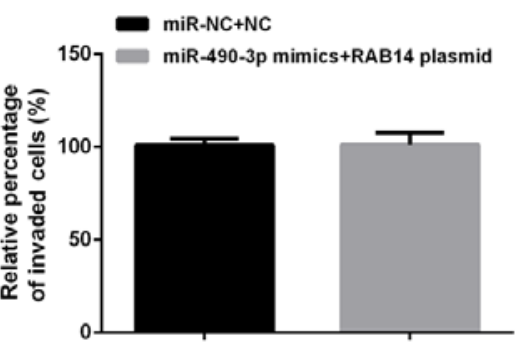

Figure 6. Effects of rescuing miR-490-3p ectopic expression by simultaneous overexpression of RAB14. (A) Cell proliferation was assessed in SW480 cells at $1,2,3,4$ and 5 days post-transfection. (B) SW480 cell invasion across an $8-\mu \mathrm{m}$ pore size membrane containing Matrigel (magnification, $\mathrm{x} 200$ ). miR, miRNA; $\mathrm{NC}$, negative control. 


\section{Discussion}

The biological roles of many miRNAs have been described in malignancy, revealing that they might function as oncogenes or tumor suppressors $(25,26)$. The present study assessed miR-490-3p, which was reported to be aberrantly expressed in various malignancies (17-20). However, the roles of miR-490-3p in tumorigenesis and cell growth are unclear.

In the present study, the clinicopathological importance of miR-490-3p in CRC was first assessed. The mean miR-490-3p expression in CRC samples were markedly reduced compared with paired adjacent non-cancerous specimens. In addition, miR-490-3p levels in patients with CRC were correlated with the pathological stage and lymph node metastasis. Furthermore, low miR-490-3p expression was associated with poor survival in CRC. As the sample size in the present study was small, additional studies with larger sample sizes are required to confirm the clinical and prognostic values of miR-490-3p in CRC. Additionally, Cox's multivariate analysis revealed distant metastasis as an independent predictive factor of overall survival in CRC.

The overexpression of miR-490-3p markedly suppressed the proliferation and colony formation of CRC cells, inducing cell cycle arrest at the G2/M phase. Reportedly, miR-490-3p regulated cyclin dependent kinase 1 that resulted in $\mathrm{G} 2 / \mathrm{M}$ phase arrest in ovarian cancer (19). Therefore in colorectal cancer, miR-490-3p might exert in a similar mechanism. However, in the present study, the upregulation of miR-490-3p had no effects on cell apoptosis. This difference in results might be because the effects of miRNA and its diverse downstream targets are dependent on the type of cancer.

Zhang et al (16) revealed that miR-490-3p promoted the growth and metastasis of hepatocellular carcinoma cells by repressing cell apoptosis. By contrast, Shen et al (17) reported that miR-490-3p targeted regulator of chromatin subfamily D member 1 (SMARCD1) to stimulate apoptosis in gastric cancer. Moreover, the in vivo experiments in the present study firstly confirmed miR-490-3p upregulation markedly inhibited the growth of CRC xenograft tumors in nude mice. These findings strongly indicated that miR-490-3p inhibited tumorigenesis in CRC.

Metastasis results from a multistep-process with cancer cells that respond to multiple intrinsic and extrinsic stimuli, detach from the primary tumor, invade the contiguous stroma, migrate to distant sites and colonize different organs $(27,28)$. Among distortions in the epigenome, aberrant expression or function of miRNAs considerably contributes to metastasis (29,30). An increasing number of studies involving preclinical models of different human tumor types indicated that specific miRNAs have functions in various steps of CRC metastasis (31-33). Therefore, how miRNAs affect CRC metastasis might provide a basis for developing new therapeutics for CRC. As aforementioned, miR-490-3p was closely related to TNM stage and lymph node metastasis. In addition, the overexpression of miR-490-3p resulted in significant repression of invasive ability in CRC cells. These findings suggested that restoring miR-490-3p in CRC might represent a novel therapeutic approach for CRC, particularly in metastatic cases.

The context-dependent roles of miRNAs in various types of cancer are likely to depend on the signaling pathways targeted. miR-490-3p was predicted to target numerous cancerassociated genes, including SMARCD1 in gastric cancer (17), TNKS2 in breast cancer (20), HMGA2 in osteosarcoma (21) and ABCC2 in ovarian cancer (34).

Notably, RAB14 was also predicted to be a target of miR-490-3p. RAB14 belongs to the RAB family of proteins. RAB14 is involved in intracellular vesicle trafficking as well as regulating signal transduction and recycling of diverse membrane receptors (35). RAB14 has a role in the progression of human cancer; however, its biological significance in CRC is largely unknown. The present study assessed the importance of RAB14 in individuals with CRC. Survival analysis indicated no significant difference between the high and low RAB14 expression groups. However, overall survival was prolonged in CRC cases with reduced RAB14 levels. Consistent with these findings, the downregulation of RAB14 in cancer cells suppressed cell proliferation and invasion as observed following the overexpression of miR-490-3p. These results confirmed that RAB14 promotes the progression of CRC.

Finally, whether RAB14 was directly targeted by miR-490-3p was examined. The ectopic expression of miR-490-3p induced marked RAB14 downregulation at the mRNA and protein levels. In addition, in CRC tissues, a low expression level of miR-490-3p was observed with a high expression level of RAB14. Conversely, a high expression level of miR-490-3p was detected with decreased RAB14 in colorectal normal tissues. These findings supported that RAB14 was a direct target of miR-490-3p and that the downregulation of RAB14 constitutes a mechanism by which miR-490-3p suppresses tumor growth.

To further test this hypothesis, a rescue experiment was carried out. The overexpression of RAB14 markedly reversed miR-490-3p-induced inhibition of proliferation and invasion in SW480 cells. However, due to distinct differences in body structures and functions of cells between animals and humans, mouse xenograft models in the present study work do not closely reflect clinical CRC, notably concerning metastasis. Therefore, further in vivo investigations are warranted to clarify the effects of miR-490-3p on the metastasis of CRC.

Recently, Xu et al (22) and Zheng et al (23) reported that miR-490-3p inhibits the metastatic and aggressive phenotype in colorectal cancer by targeting transforming growth factor (TGF)- $\beta$ and Wnt/ $\beta$-catenin signaling pathways. TGF- $\beta$, a cytokine with multiple biological effects, was first reported as an inducer of epithelia-mesenchymal transition (EMT) in normal mammary epithelial cells. Several subsequent studies reported important roles of TGF- $\beta$-induced EMT in tumor metastasis, including colorectal cancer (36). The canonical Wnt signaling pathway is aberrantly activated in the majority of patients with CRC due to mutations in adenomatous polyposis coli or $\beta$-catenin, and it was demonstrated that the hyperactivation of Wnt signaling has an essential role in the pathogenesis of CRC $(37,38)$. Recent studies have indicated that TGF- $\beta$ and TGF $\beta$-induced factor homeobox 1 were able to activate the $\mathrm{Wnt} / \beta$-catenin signaling pathway in colorectal cancer (39), breast cancer (40) and systemic sclerosis (41). Hou et al (5) revealed that the Wnt inhibitor abolished the effect of RAB14 on cell proliferation and Wnt target genes 
in ovarian cancer, demonstrating that $\mathrm{RAB} 14$ regulated cell aggressiveness via the $\mathrm{Wnt} / \beta$-catenin pathway. Furthermore, RAB family protein, RAB11, with similar structure to RAB14, was reported to directly regulate the recycling of TGF- $\beta$ receptors irrespective of the activation state of the receptors (42). Therefore, based on these recent studies, RAB14 might be an upstream key factor in regulating, or at least, affecting the functions of TGF- $\beta$ and the $\mathrm{Wnt} / \beta$-catenin signaling pathway. In the present study, miR-490-3p targeted RAB14 to affect the biological functions of colorectal cancer. RAB14 might be the main target of miR-490-3p in regulating the malignant phenotype of colorectal cancer, which remains to be verified in further investigations.

In summary, the present study indicated that human CRC tissue samples and cells have decreased miR-490-3p expression compared with colorectal normal tissues and cells respectively, which was also reflected by a poor prognosis of patients with CRC. Meanwhile, miR-490-3p suppressed tumorigenesis and aggressiveness in CRC by directly targeting RAB14. These findings provide novel insights into targeting miR-490-3p/ RAB14 interactions as a therapeutic option for CRC.

\section{Acknowledgements}

Not applicable.

\section{Funding}

The present study was supported by grants from the National Natural Science Foundation of China (grant nos. 81702354, 81672375 and 81572379).

\section{Availability of data and materials}

The analyzed datasets generated during the study are available from the corresponding author on reasonable request.

\section{Authors' contributions}

BW, YY and ZS were responsible for the study design, drafting and editing of the original article, data acquisition and data analysis. BW, CC and $\mathrm{HJ}$ performed the experiments. MY, KJ and $\mathrm{SW}$ were responsible for data acquisition and analysis. MY, CC and KJ were responsible for data interpretation and methodology. YY, ZS and SW were responsible for supervision. BW, MY, YY and ZS revised the manuscript. All authors have read and approved the final manuscript.

\section{Ethics approval and consent to participate}

The animal studies were conducted according to the recommendations outlined in the Guide for the Care and Use of Laboratory Animals in the Weatherall report. The animal experiments were approved by the Animal Research Committee of the Peking University People's Hospital (Beijing, China).

\section{Patient consent for publication}

Written consent for research and publication was obtained from each patient involved in this study.

\section{Competing interests}

The authors declare that they have no competing interests.

\section{References}

1. Siegel RL, Miller KD and Jemal A: Cancer Statistics, 2017. CA Cancer J Clin 67: 7-30, 2017.

2. Fakih MG: Metastatic colorectal cancer: Current state and future directions. J Clin Oncol 33: 1809-1824, 2015.

3. Stenmark H: Rab GTPases as coordinators of vesicle traffic. Nat Rev Mol Cell Biol 10: 513-525, 2009.

4. Reiner DJ and Lundquist EA: Small GTPases. WormBook 1.67.2.: $1-99,2016$.

5. Hou R, Jiang L, Yang Z, Wang S and Liu Q: Rab14 is overexpressed in ovarian cancers and promotes ovarian cancer proliferation through Wnt pathway. Tumour Biol 37: 16005-16013, 2016.

6. Sun J, Feng X, Gao S and Xiao Z: microRNA-338-3p functions as a tumor suppressor in human non-small-cell lung carcinoma and targets Ras-related protein 14. Mol Med Rep 11: 1400-1406, 2015.

7. Yu J, Wang L, Yang H, Ding D, Zhang L, Wang J, Chen Q, Zou Q, Jin Y and Liu X: Rab14 suppression mediated by miR-320a inhibits cell proliferation, migration and invasion in breast cancer. J Cancer 7: 2317-2326, 2016.

8. Li Y, Liu H, Shao J and Xing G: miR-320a serves as a negative regulator in the progression of gastric cancer by targeting RAB14. Mol Med Rep 16: 2652-2658, 2017.

9. Guo B, Wang W, Zhao Z, Li Q, Zhou K, Zhao L, Wang L, Yang J and Huang C: Rab14 act as oncogene and induce proliferation of gastric cancer cells via AKT signaling pathway. PLoS One 12: e0170620, 2017.

10. Kasinski AL and Slack FJ: Epigenetics and genetics. MicroRNAs en route to the clinic: Progress in validating and targeting microRNAs for cancer therapy. Nat Rev Cancer 11: 849-864, 2011.

11. Valeri N, Croce CM and Fabbri M: Pathogenetic and clinical relevance of microRNAs in colorectal cancer. Cancer Genomics Proteomics 6: 195-204, 2009.

12. Shen Z, Zhou R, Liu C, Wang Y, Zhan W, Shao Z, Liu J, Zhang F, $\mathrm{Xu}$ L, Zhou X, et al: MicroRNA-105 is involved in TNF- $\alpha$ related tumor microenvironment enhanced colorectal cancer progression. Cell Death Dis 8: 3213, 2017.

13. Shen ZL, Wang B, Jiang KW, Ye CX, Cheng C, Yan YC, Zhang JZ, Yang Y, Gao ZD, Ye YJ, et al: Downregulation of miR-199b is associated with distant metastasis in colorectal cancer via activation of SIRT1 and inhibition of CREB/KISS1 signaling. Oncotarget 7: 35092-35105, 2016.

14. Wang B, Shen ZL, Gao ZD, Zhao G, Wang CY, Yang Y, Zhang JZ, Yan YC, Shen C, Jiang KW, et al: miR-194, commonly repressed in colorectal cancer, suppresses tumor growth by regulating the MAP4K4/c-Jun/MDM2 signaling pathway. Cell Cycle 14: 1046-1058, 2015

15. Wang B, Shen ZL, Jiang KW, Zhao G, Wang CY, Yan YC, Yang Y, Zhang JZ, Shen C, Gao ZD, et al: MicroRNA-217 functions as a prognosis predictor and inhibits colorectal cancer cell proliferation and invasion via an AEG-1 dependent mechanism. BMC Cancer 15: 437, 2015.

16. Zhang LY, Liu M, Li X and Tang H: miR-490-3p modulates cell grow th and epithelial to mesenchymal transition of hepatocellular carcinoma cells by targeting endoplasmic reticulum-Golgi intermediate compartment protein 3 (ERGIC3). J Biol Chem 288: 4035-4047, 2013

17. Shen J, Xiao Z, Wu WK, Wang MH, To KF, Chen Y, Yang W, Li MS, Shin VY, Tong JH, et al: Epigenetic silencing of miR-490-3p reactivates the chromatin remodeler SMARCD1 to promote Helicobacter pylori-induced gastric carcinogenesis. Cancer Res 75: 754-765, 2015.

18. Qu M, Li L and Zheng WC: Reduced miR-490-3p expression is associated with poor prognosis of Helicobacter pylori induced gastric cancer. Eur Rev Med Pharmacol Sci 21: 3384-3388, 2017.

19. Chen S, Chen X, Xiu YL, Sun KX and Zhao Y: MicroRNA-490-3P targets CDK1 and inhibits ovarian epithelial carcinoma tumorigenesis and progression. Cancer Lett 362: 122-130, 2015.

20. Jia Z, Liu Y, Gao Q, Han Y, Zhang G, Xu S, Cheng K and Zou W: miR-490-3p inhibits the growth and invasiveness in triplenegative breast cancer by repressing the expression of TNKS2 . Gene 593: 41-47, 2016 
21. Liu W, Xu G, Liu H and Li T: MicroRNA-490-3p regulates cell proliferation and apoptosis by targeting HMGA2 in osteosarcoma. FEBS Lett 589B: 3148-3153, 2015.

22. Xu X, Chen R, Li Z, Huang N, Wu X, Li S, Li Y and Wu S: MicroRNA-490-3p inhibits colorectal cancer metastasis by targeting TGF $\beta R 1$. BMC Cancer 15: 1023, 2015.

23. Zheng K, Zhou X, Yu J, Li Q, Wang H, Li M, Shao Z, Zhang F, Luo Y, Shen Z, et al: Epigenetic silencing of miR-490-3p promotes development of an aggressive colorectal cancer phenotype through activation of the Wnt/ $\beta$-catenin signaling pathway. Cancer Lett 376: 178-187, 2016.

24. Livak KJ and Schmittgen TD: Analysis of relative gene expression data using real-time quantitative PCR and the 2(-Delta Delta C(T)) Method. Methods 25: 402-408, 2001.

25. Bracken CP, Scott HS and Goodall GJ: A network-biology perspective of microRNA function and dysfunction in cancer. Nat Rev Genet 17: 719-732, 2016.

26. Rupaimoole R, Calin GA, Lopez-Berestein G and Sood AK: miRNA deregulation in cancer cells and the tumor microenvironment. Cancer Discov 6: 235-246, 2016.

27. Profumo V and Gandellini P: MicroRNAs: Cobblestones on the road to cancer metastasis. Crit Rev Oncog 18: 341-355, 2013.

28. Shen Z, Wang B, Luo J, Jiang K, Zhang H, Mustonen H, Puolakkainen P, Zhu J, Ye Y and Wang S: Global-scale profiling of differential expressed lysine acetylated proteins in colorectal cancer tumors and paired liver metastases. J Proteomics 142: 24-32, 2016.

29. Doldi V, Pennati M, Forte B, Gandellini P and Zaffaroni N: Dissecting the role of microRNAs in prostate cancer metastasis: Implications for the design of novel therapeutic approaches. Cell Mol Life Sci 73: 2531-2542, 2016

30. White NM, Fatoohi E, Metias M, Jung K, Stephan C and Yousef GM: Metastamirs: A stepping stone towards improved cancer management. Nat Rev Clin Oncol 8: 75-84, 2011.

31. Gandellini P, Doldi V and Zaffaroni N: microRNAs as players and signals in the metastatic cascade: Implications for the development of novel anti-metastatic therapies. Semin Cancer Biol 44: 132-140, 2017.

32. Hur K, Toiyama Y, Okugawa Y, Ide S, Imaoka H, Boland CR and Goel A: Circulating microRNA-203 predicts prognosis and metastasis in human colorectal cancer. Gut 66: 654-665, 2017.
33. Chen DL, Wang ZQ, Zeng ZL, Wu WJ, Zhang DS, Luo HY, Wang F, Qiu MZ, Wang DS, Ren C, et al: Identification of microRNA-214 as a negative regulator of colorectal cancer liver metastasis by way of regulation of fibroblast growth factor receptor 1 expression. Hepatology 60: 598-609, 2014.

34. Tian J, Xu YY, Li L and Hao Q: miR-490-3p sensitizes ovarian cancer cells to cisplatin by directly targeting ABCC2. Am J Transl Res 9: 1127-1138, 2017.

35. Takai Y, Sasaki T and Matozaki T: Small GTP-binding proteins. Physiol Rev 81: 153-208, 2001.

36. Jung B, Staudacher JJ and Beauchamp D: Transforming growth factor $\beta$ superfamily signaling in development of colorectal cancer. Gastroenterology 152: 36-52, 2017.

37. Morin PJ, Sparks AB, Korinek V, Barker N, Clevers H, Vogelstein B and Kinzler KW: Activation of beta-catenin-Tcf signaling in colon cancer by mutations in beta-catenin or APC. Science 275: 1787-1790, 1997.

38. Kinzler KW and Vogelstein B: Lessons from hereditary colorectal cancer. Cell 87: 159-170, 1996.

39. Wang JL, Qi Z, Li YH, Zhao HM, Chen YG and Fu W: TGF $\beta$ induced factor homeobox 1 promotes colorectal cancer development through activating Wnt/ $\beta$-catenin signaling. Oncotarget 8: 70214-70225, 2017.

40. Ma F, Li W, Liu C, Li W, Yu H, Lei B, Ren Y, Li Z, Pang D and Qian C: miR-23a promotes TGF- $\beta 1$-induced EMT and tumor metastasis in breast cancer cells by directly targeting $\mathrm{CDH} 1$ and activating Wnt/ $\beta$-catenin signaling. Oncotarget 8: 69538-69550, 2017.

41. Gillespie J, Ross RL, Corinaldesi C, Esteves F, Derrett-Smith E, McDermott MF, Doody GM, Denton CP, Emery P and Del Galdo F: Transforming growth factor $\beta$ activation primes canonical Wnt signaling through down-regulation of Axin-2. Arthritis Rheumatol 70: 932-942, 2018.

42. Mitchell H, Choudhury A, Pagano RE and Leof EB: Liganddependent and -independent transforming growth factor-beta receptor recycling regulated by clathrin-mediated endocytosis and Rab11. Mol Biol Cell 15: 4166-4178, 2004. 